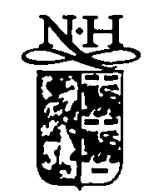

ELSEVIER

\title{
Magnetic-field dependent Fermi surfaces in quasi-2D organic conductors
}

\author{
J. Singleton ${ }^{\text {a } *}$, A.A. House ${ }^{a}$, N. Harrison ${ }^{b}$, I. Deckers ${ }^{\text {b }}$, S.J. Blundell ${ }^{\text {a }}$, \\ J.A.A.J. Perenboom ${ }^{\text {c }}$, A. Polisskii a, W. Hayes ", F. Herlach b , M. Kurmoo d, P. Day d \\ - University of Oxford Department of Physics, The Clarendon Laboratory, Parks Road, Oxford OX1 3PU, UK \\ baboratorium voor Vaste-Stoffysica en Magnetisme, Katholieke Universiteit Leuven, Celestijnenlaan 200 D, B-3030 Lewven, Belgium \\ - High Field Magnet Laboratory and Research Institute for Materials, 6525 EDNijmegen, The Netherlands \\ d The Royal Institution, 21 Albemarle Street, London W1X 4BS, UK
}

Received 1 August 1995; accepted for publication 5 September 1995

\begin{abstract}
We review rocent studies of charge-transfer salts of the ion bis(ethylenedithio)tetrathiafulvalene which exhibit spin-density wave ground states, concentrating on techniques involving high magnetic fields and experiments carried out by the Oxford group and coworkers.
\end{abstract}

Keywords: Anglo-dependent magnetoresistance oscillations; BEDT-TTF; Charge-transfer salts; De Haas-van Alphen; Magnetotransport; Organic molecular metals

\section{Introduction}

Traditionally, fundamental solid-state physics has almost exclusively involved the study of inorganic elements, alloys, and simple compounds, the underlying assumption being that fundamental physics research is most usefully concentrated on chemically simple materials. Our recent work is built upon the realisation that such an assumption is quite misplaced, that in fact some of the most exciting studies in solid-state physics can and should be attempted on organic materials which

* Corresponding author. Fax: 441865 272400, e-mail: jsingle@vax.ox.ac.uk are chemically very complicated. The attraction of organic molecular systems is that though chemically complex, in other ways they are beautifully simple and can often provide much information about basic phenomena, like superconductivity and magnetism ([1-3] and references therein). As an example of this, charge-transfer salts of the ion bis(ethylenedithio) tetrathiafulvalene (ET or BEDT-TTF) form a versatile and interesting system for the study of many problems in solidstate physics. By varying the anion $\mathrm{X}$ incorporated into the charge-transfer salt (ET) $\mathrm{X}$, the stoichiometry and band-filling may be adjusted, producing compounds which are metallic, semimetallic, semiconducting or superconducting [1-3]. The chargetransfer salts have a very simple quasi-two dimen- 
sional (q2D) bandstructure and a well-defined Fermi surface, and single crystals of high quality are available, making them amenable to bandstructure characterisation techniques such as the Shubuikov-de Haas (SdH) effect.

In previous reviews $[2,3]$, we have concentrated on some of the similarities between the ET salts and semiconductor systems such as superlattices. In this paper, we shall instead describe some of the contrasts between the two systems, and focus on two experimental techniques which are now regularly applied in the study of organic molecular metals, but not semiconductors.

\section{Field-dependent fermi surfaces}

\subsection{The de Haas-van Alphen effect up to $54 T$}

The areal carrier densities in ET salts are $100-1000$ times higher than those in semiconductor heterostructures, so that many interactions which are of negligible size in the latter systems exert a major influence. In particular, carrier-phonon and carrier-carrier interactions are strong, often resulting in heavy effective masses $\left(1.5-10 \mathrm{~m}_{\mathrm{o}}\right)$, large densities of states at the Fermi energy, and superconducting or spin-density wave (SDW) ground states $[1,3]$. Furthermore, typical laboratory magnetic fields cause significant perturbations of the bandstructure and can result in field-induced changes in the Fermi surface [4-6].

These points are illustrated by the salts $\alpha-(\mathrm{ET})_{2} \mathbf{M H g}(\mathrm{NCS})_{4}\left(\mathrm{M}=\mathrm{NH}_{4}, \quad \mathrm{~K}, \quad \mathrm{Rb}, \quad \mathrm{Tl}\right)$. Although all possess very similar calculated Fermi surfaces (Fig. 1a, inset) [7], consisting of an open $1 \mathrm{D}$ electron section and a closed $2 \mathrm{D}$ hole pocket, the salt with $\mathrm{M}=\mathrm{NH}_{4}$ is a superconductor below $1 \mathrm{~K}$, whilst those with $\mathrm{M}=\mathrm{K}, \mathrm{Tl}, \mathrm{Rb}$ exhibit SDW ground states at low temperature (see Refs. [4-6] and references therein). When an SDW forms, a so-called "nesting vector" in $k$-space translates one side of the 1D Fermi surface into the other, thereby opening up a band gap and lowering the energy of the system [8]. The nesting vector introduces a new periodicity in the crystal which results in a smaller Brillouin zone. In turn, the new zone boundaries "cut and paste" any remaining pieces
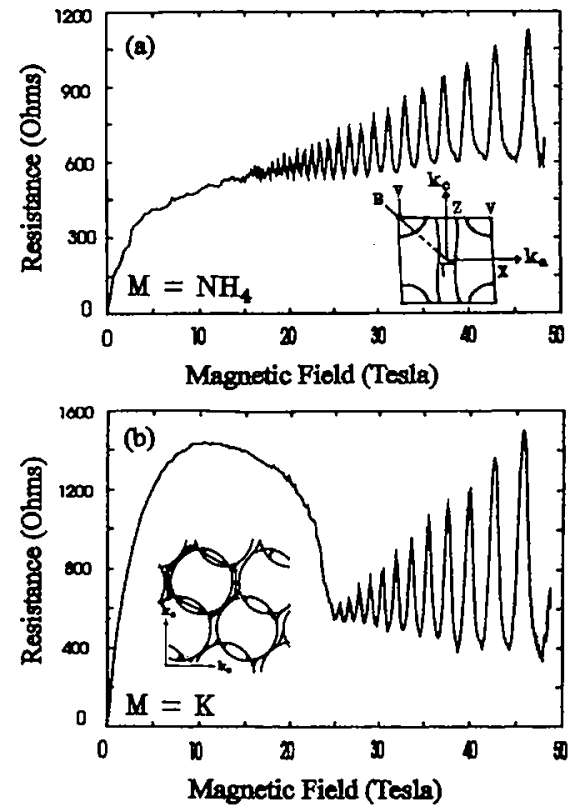

Fig. 1. Magnetoresistance of $\alpha-(\mathrm{ET})_{2} \mathrm{MHg}(\mathrm{NCS})_{4}$ at $T \approx 1 \mathrm{~K}$ for (a) $\mathrm{M}=\mathrm{NH}_{4}$ and (b) $\mathrm{M}=\mathrm{K}$. The insets show (a) the calculated Fermi surface and (b) a proposed Fermi surface in the SDW state.

of Fermi surface into a new arrangement. Additional small pockets of Fermi surface may occur due to imperfect nesting of the $1 \mathrm{D}$ parts. Such a proposed reconstruction of the Fermi surface for $\alpha-(\mathrm{ET})_{2} \mathrm{MHg}(\mathrm{NCS})_{4}(\mathrm{M}=\mathrm{Tl}, \mathrm{K})$ due to an SDW [9] is shown as an inset to Fig. 1b.

One of the most notable features of the $M=K$, $\mathbf{R b}$ and Tl salts is observed as a sharp kink in the magnetoresistance (MR) (Fig. 1b); this has become known as the "kink transition". The kink occurs at around $23 \mathrm{~T}$ in the $\mathrm{M}=\mathrm{K}$ salt, and is thought to represent destruction of the SDW ground state (i.e. a transition from a Fermi surface possibly like the one in Fig. 1b to that in Fig. 1a; Refs. [4-6] and references therein). The kink is absent in the $\mathbf{M}=\mathrm{NH}_{4}$ salt (c.f. Figs. $1 \mathrm{a}$ and $1 \mathrm{~b}$ ).

Recently we have studied the de Haas-van Alphen (dHvA) effect (i.e. oscillatory magnetisation) of $\alpha-(\mathrm{ET})_{2} \mathrm{KHg}(\mathrm{NCS})_{4}$ in pulsed magnetic fields of up to $54 \mathrm{~T}$, in order to clarify the fieldinduced changes in bandstructure occurring at the kink [10]. Fig. 2a shows a typical dHvA signal from a single $1 \mathrm{~mm} \times 0.5 \mathrm{~mm} \times 0.1 \mathrm{~mm}$ crystal of 

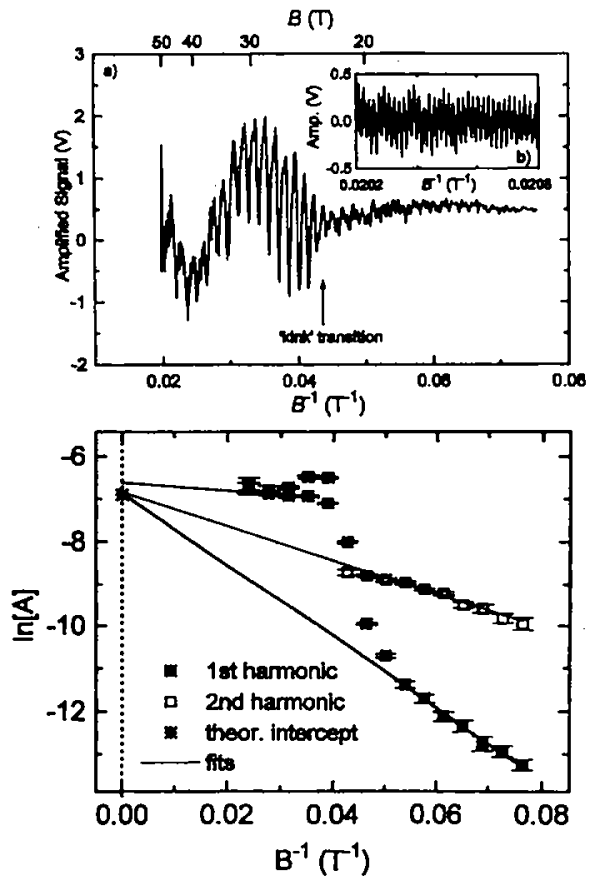

Fig. 2. (a) dHvA oscillations for $\alpha-(\mathrm{ET})_{2} \mathrm{KHg}(\mathrm{NCS})_{4}$ at $350 \mathrm{mK}$. (b) Enlargement of the high-field region, showing $\mathrm{Cu} \mathrm{dHvA}$. (Bottom) Dingle plots for the first and second harmonics of the dHvA oscillations.

$\alpha$-(ET) ${ }_{2} \mathrm{KHg}(\mathrm{NCS})_{4}$, recorded using a compensated pick-up coil technique (a direct measurement of $\mathrm{d} M / \mathrm{d} B$ ). The data were measured on the rising side of the field pulse; higher $\mathrm{dB} / \mathrm{d} t$ produces a better signal-to-noise ratio, allowing $\mathrm{dHvA}$ oscillations at fields below the kink to be clearly resolved. The dHvA oscillations above $23 \mathrm{~T}$ are attributed to the 2D hole pocket of the Fermi surface shown in Fig. 1a. Note that the oscillations for fields below the kink exhibit the same frequency $(F=$ $670 \pm 3 \mathrm{~T}$ ), but with a stronger second harmonic component. The apparent increase in noise towards peak field is partly due to copper-belly $\mathrm{dHvA}$ originating from the copper wire of the detection coils (Fig. 2b), illustrating the sensitivity of the technique [10].

The importance of using the highest possible fields is illustrated by Fig. 2, bottom, which shows "Dingle plots" (i.e. logarithmic corrected dHvA oscillation amplitudes versus $1 / B$ [11]) for the first and second harmonics of the $670 \mathrm{~T}$ series. The new feature revealed using high fields is the region of the Dingle plot with a very small gradient, corresponding to $B>\sim 27 \mathrm{~T}$. Earlier studies up to $\sim 30$ $T$ had only accessed the steeply sloping middle and flatter low-field regions of the Dingle plots $[4,6]$, and the authors had incorrectly taken this to imply that the scattering rate at high fields increased. However, it is apparent from Fig. 2, bottom, that the gently sloping region corresponding to $B>\sim 27 \mathrm{~T}$ represents the true behaviour of the high-field state; the other shallow sloped region $(B<\sim 20 \mathrm{~T})$ corresponds to the low-field state. The region between can therefore be considered as a gradual transition between the two regimes, and is roughly centred on the kink at $\sim 23 \mathrm{~T}$. The close correlation between the infinite field intercepts above and below the kink, and the fact that the dHvA frequency $F$ is the same above and below the kink, suggest that the quantum oscillations detected in both regimes originate from similar, if not identical, pieces of Fermi surface. However, the quasi-particle scattering rate increases greatly from $\tau^{-1} \approx 2 \times 10^{11} \mathrm{~s}^{-1}$ above $\sim 27 \mathrm{~T}$ to $\tau^{-1} \approx 2 \times 10^{12} \mathrm{~s}^{-1}$ below $\sim 20 \mathrm{~T}$, indicating that additional scattering processes occur at fields below the kink, probably due to the formation of the SDW [10].

The temperature dependence of data such as those in Fig. 2a may be analysed to yield effective masses $m^{*}$ for the 2D carriers. The availability of data up to $\sim 54 \mathrm{~T}$ allows reliable values to be obtained at fields well above the kink. Values of $m^{*} \approx 1.5 m_{0}$ were obtained for B $<20 \mathrm{~T}$, whilst for B $>27 \mathrm{~T}, m^{*} \approx 2.7 m_{\mathrm{e}}$. This large increase probably signals an enhancement of the carrier-carrier and/or carrier-phonon interactions above the kink. It is notable that the effective mass of the $2 \mathrm{D}$ holes in $\alpha-(\mathrm{ET})_{2} \mathrm{KHg}(\mathrm{NCS})_{4}$ measured at fields below the kink is anomalously low compared to those of isostructural salts (e.g. $\left.\alpha-(\mathrm{ET})_{2} \mathrm{NH}_{4} \mathrm{Hg}(\mathrm{NCS})_{4}[12]\right)$ which do not exhibit a SDW ground state. The larger mass in the highfield state may therefore be a more representative value.

Finally, we note that the dHvA oscillations at fields above $30 \mathrm{~T}$ contain unusually large contributions from higher harmonics [10]. These higher harmonics do not obey the Lifschitz-Kosevich formula, which successfully predicts the behaviour 
of "conventional" metals. Such anomalous behaviour and its cause is currently a focus for study in the organic metals [13-15]. The utility of the dHvA pick-up coil technique in this context cannot be overemphasised; in contrast to either $\mathrm{SdH}$ $[13,14]$ or torque $\mathrm{dHvA}$ measurements $[15]$, the signal derived from the sample may be simply related in a quantitative manner to theoretical expressions for the density of states at the Fermi energy [10]. In addition, its sensitivity is now sufficient to study some semiconductor systems.

\subsection{Angle-dependent magnetoresistance oscillations}

We have glibly thus far been referring to the sections of Fermi surface in the ET salts as "1D" and " $2 \mathrm{D}$ ". In fact, there is a small contribution to the bandstructure from molecular overlaps in the interplane direction, leading to slightly warped or corrugated q1D and q2D Fermi surfaces. The SdH and dHvA effects tend not to pick up any information about this warping, because each frequency observed is proportional to the area of an extremal orbit of the Fermi surface [11]. Furthermore, the warping of the q2D sections is in fact small, so that separate neck and belly orbits are rarely seen [2]. The orbits which are not extremal do not contribute to this oscillatory signal but give rise to e.g. the non-oscillatory background MR. However, this background MR can depend quite dramatically on the direction of the applied magnetic field, and can give information about Fermi surface warping. The effect has been exploited in the technique known as "angle-dependent magnetoresistance oscillations" (AMRO), now commonly used to characterise the Fermi surfaces of chargetransfer salts. AMRO can be observed at much higher temperatures and in much lower applied fields than either the SdH or dHvA effects [1]. The latter arise due to well-resolved Landau levels moving through a sharply defined Fermi surface, and this restriction does not apply so stringently to AMRO. The information obtained from AMRO can therefore be complementary to $\mathrm{SdH}$ and $\mathrm{dHvA}$ effects since it is due to all carriers on the Fermi surface, and not just those performing extremal orbits.

Semi-classical calculations of AMRO can be performed using the Boltzmann transport equation, an integral (over all states at the Fermi surface) of the velocity-velocity correlation function for each Fermi surface orbit [16]. This can change dramatically as the direction of the magnetic field is changed, altering the paths of all the Fermi surface orbits. For q2D Fermi surfaces, this manifests itself as the Yamaji effect $[17,18]$; as the field is tilted away from the axis of the warped cylindrical Fermi surface, MR oscillations can be observed which can be directly related to the degree of warping. In order to apply such models to AMRO in substances like $\alpha-(\mathrm{ET})_{2} \mathrm{KHg}(\mathrm{SCN})_{4}$, one must also include the effect of the q1D part of the Fermi surface. This can be considered to be two slightly warped sheets with a dispersion relation given by

$E(\mathbf{k})=\hbar v_{F}\left(\left|k_{x}\right|-k_{F}\right)-\sum_{m, n} t_{m n} \cos \left(m b k_{y}+n c k_{z}\right)$,

where the parameters $t_{m n}$ are the Fourier components of the corrugation of the Fermi sheets [16].

Turning to experimental data, Fig. 3 shows the MR of $\alpha-(E T)_{2} \mathrm{KHg}(\mathrm{NCS})_{4}$ as a function of the angle $\theta$ between the normal to the 2D plane and the field [4]. The azimuthal angle $\theta$ in Fig. 3 describes the plane of rotation of the magnetic field. At $15 \mathrm{~T}$, the AMRO appear as sharp dips in the MR periodic in $\tan (\theta)$, and the period is proportional to $1 / \cos \left(\phi-\phi_{0}\right)$, with $\phi_{0} \approx 21^{\circ}$. Such AMRO are characteristic of a q1D section of Fermi surface inclined at $\sim 21^{\circ}$ to the crystallographic $b^{*} c$ plane. At $24 \mathrm{~T}$, the AMRO appear instead as broader peaks which do not exhibit a strong $\phi$ dependence; this is the characteristic behaviour of the Yamaji oscillations caused by 2D sections of the Fermi surface $[4,6]$.

The AMRO clearly sense a Fermi surface reconstruction in this material as the kink field is exceeded. We have already seen that above the kink field, the Fermi surface is thought to contain both q1D and q2D sections (Fig. 1a), whilst below the kink field, q2D sections must occur for the dHvA and SdH effects to be observed (see Fig. 2a) [4], in addition to the q1D sections which dominate the AMRO. If both types of Fermi surface occur above and below the kink, why is a 

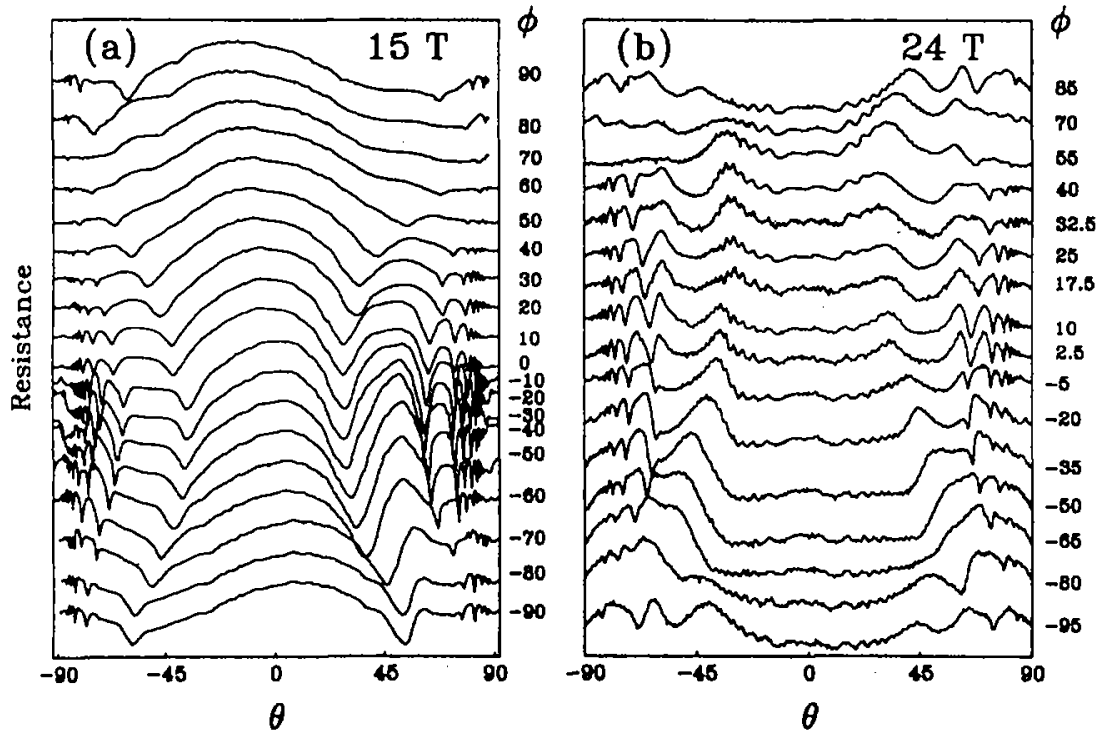

Fig. 3. Angle-dependent magnetoresistance oscillations for $\alpha-(\mathrm{ET})_{2} \mathrm{KHg}(\mathrm{NCS})_{4}$ at $1.5 \mathrm{~K}$ for several values of the angle $\phi$ at an applied field of (a) 15 and (b) $24 \mathrm{~T}$.

transition in the AMRO observed? A second problem concerns the parameters $t_{m n}$. These are often related to the transfer integrals associated with the lattice vector $r_{m n}=(0, m b, n c)$ [19]. Simulations of AMRO seen at low fields in $(\mathrm{ET})_{2} \mathrm{KHg}(\mathrm{SCN})_{4}$ require these to decay relatively slowly with increasing $m$ and $n$ in order to model the data $[4,16,20]$, whereas one might expect anything other than the nearest-neighbour and next-nearest neighbour overlaps to be negligibly small [8]. This is in fact why no AMRO due to q1D sections of the Fermi surface are generally observed in other ET salts, and in particular, why none is observed above the kink transition in $(\mathrm{ET})_{2} \mathrm{KHg}(\mathrm{SCN})_{4}$ (Fig. 4b). Thus, only q2D AMRO is observed above the kink. However, below the kink, the nesting of the Fermi surface removes the weakly corrugated q1D sections of Fermi surface and "cuts up and glues together" pieces of q2D Fermi surface. The reconstructed Fermi surface consists of a very highly and irregularly corrugated Fermi sheet (see Fig. 1b for a possible candidate) since it consists of a periodic assembly of cylindrical sections; the corrugation thus has a high harmonic content. Therefore the $t_{m x}$ are not transfer integrals so much as Fourier components of the corrugation.

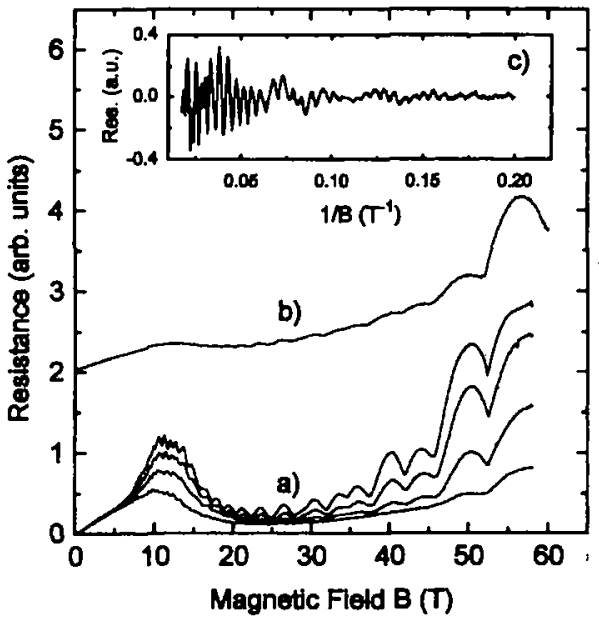

Fig. 4. MR of $\beta^{\prime \prime}-(E T)_{2} \mathrm{AuBr}_{2}$. (a) Top to bottom, $490,690,960$ $\mathrm{mK}$ and $1.44 \mathrm{~K}$ data (b) Data for a lower quality sample $(380$ $\mathrm{mK}$ ). (c) Oscillatory component of $\mathrm{MR}$ in (a) at $490 \mathrm{mK}$.

Although some q2D pockets are also formed in the reconstructed Fermi surface, these do not contribute to the AMRO (perhaps because they themselves are irregularly corrugated, and the q2D "Yamaji" AMRO effect relies on cylindrical sections of Fermi surface with weak and regular 
corrugation). The AMRO in this q1D case arises from the fact that for any Fourier component of corrugation, the velocity is more effectively averaged when electrons are not travelling along the axis of the corrugation than when they are; thus sharp resistance minima are obtained when the orbits run along a Fourier component of the corrugation.

\subsection{Another example}

Fig. 4 shows the MR of $\beta^{\prime \prime}-(\mathrm{ET})_{2} \mathrm{AuBr}_{2}$, recorded using a pulsed magnet and a $10 \mu \mathrm{A}, 250 \mathrm{kHz} \mathrm{AC}$ current [21]. The Fermi surface calculated for $\beta^{\prime \prime}-(\mathrm{ET})_{2} \mathrm{AuBr}_{2}$ only contains one closed $2 \mathrm{D}$ hole pocket, and so a single series of $\mathrm{SdH}$ oscillations is expected. However, even a cursory inspection of Fig. 4 indicates that there are many sets of oscillations present, and a Fourier transform of the entire field range shows no less than fourteen different frequencies, ranging from 38 to $414 \mathrm{~T}$. Even when obvious second harmonics have been eliminated, eight frequencies remain.

The plethora of frequencies results from the presence of an SDW, evidence for which is also seen in the spin susceptibility [22]. Four of the SdH frequencies can be explained using an SDW model [22], but the problem of accounting for the remaining four sets of oscillations remains. The answer is provided by the AMRO data in Fig. 5. Note that most of the features in the MR do not shift as $B$ is increased, but merely grow in amplitude. However, for $B>\sim 11 \mathrm{~T}$, a new series of AMRO appears, indicating that the Fermi surface has changed in form. This transition at $B \sim 11 \mathrm{~T}$ results in shifts of the SdH frequencies, and herein lies the explanation for the eight frequencies seen in the Fourier transforms of Fig. 4; four correspond to the Fermi surface below $11 \mathrm{~T}$, and the other four result from the altered Fermi surface above $11 \mathrm{~T}$. This has been confirmed by analysis of the $\mathrm{SdH}$ frequencies over restricted field ranges [21].

The smallest of the Fermi surface pockets, corresponding to a frequency of $38 \mathrm{~T}$, approaches the quantum limit $(v=2)$ at peak field. The background MR increases dramatically above $40 \mathrm{~T}$, rising by over an order of magnitude in some samples (Fig. 4a). The increase is suppressed by raising the

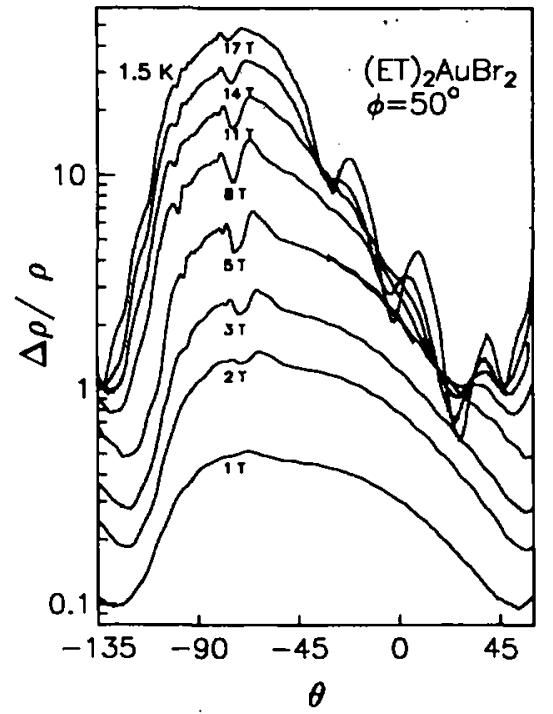

Fig. 5. AMRO data for $\beta^{\prime \prime}-(\mathrm{ET})_{2} \mathrm{AuBr}_{2}$.

temperature (Fig. 4b). Effects such as simple depopulation of a small Fermi surface pocket or destruction of the SDW ground state by the field are almost invariably expected to lead to a sharp decrease in resistance [2], rather than the increase observed experimentally. The data instead suggest that a further field-induced rearrangement of the SDW may occur at high magnetic fields. Theoretical papers have proposed that strong carrier self-energy effects will occur as a high carrierdensity 2D metal enters the ultraquantum limit, leading to an enhancement of the density of states (DOS) at the Fermi energy (e.g. Ref. [23]). It has been suggested that this could in turn promote phenomena such as re-entrant high-field superconductivity [23]. SDW groundstates often occur in competition with superconductivity in organic molecular metals $[2,3]$. It is therefore possible that one of the suggested ultraquantum limit DOS enhancement mechanisms may be initiating a further modification of the SDW state at $\sim 40 \mathrm{~T}$, rather than re-entrant superconductivity [21].

\section{Acknowledgements}

This work is supported by EPSRC, the EU and the Royal Society (UK). The Nijmegen High Field 
Magnet Laboratory is supported by FOM and the EU Large Installations Plan. We thank our many collaborators in Boston, Bristol, Cambridge, Chernogolovka, Leuven, London, Moscow, Nijmegen, Oxford, Sheffield, Tallahassee and Tsukuba for invaluable help, discussions and access to unpublished data.

\section{References}

[1] For a recent review, see Proc. Int. Conf. of Synthetic Metals, Scoul, Korea, July 1994, Synth. Met. 69-71 (1995).

[2] J. Singleton, F.L. Pratt, M. Doporto, J.M. Caulfield, S.O. Hill, T.J.B.M. Janssen, I. Deckers, G. Pitsi, F. Herlach, W. Hayes, J.A.A.J. Perenboom, M. Kurmoo and P. Day, Physica B 184 (1993) 470-480.

[3] J. Singleton, J.M. Caulfield, S.O. Hill, S.J. Blundell, W. Lubczyuski, A. House, W. Hayes, J.A.A.J. Perenboom, M. Kurmoo and P. Day, Physica B 211 (1985) 400.

[4] J. Caulfield, S.J. Blundell, M.S.L. du Croo de Jongh, P.T.J. Hendriks, J. Singleton, M. Doporto, F.L. Pratt, A. House, J.A.A.J. Perenboom, W. Hayes, M. Kurmoo and P. Day, Phys. Rev. B 51 (1995) 8325.

[5] J.S. Brooks, X. Chen, S.J. Klepper, S. Valfells, G.J. Athas, Y. Tanaks, T. Kinoshits, N. Kinoshita, M. Tokomoto, H. Anzai and C.C. Agosta, Phys. Rev. B 52 (1995) 14457.

[6] T. Sasaki and N. Toyota, Phys. Rev. B 49 (1994) 10120.

[7] H. Mori, S. Tanaka, M. Oshima, G. Saito, T. Mori, Y. Maruyama and H. Inkuchi, Bull. Chem. Soc. Jpn. 63 (1990) 2183.

[8] T. Ishiguro and K. Yamaji, Organic Superconductors (Springer, Berlin, 1990).
[9] M.V. Kartsovnik, H. Ito, T. Ishiguro, H. Mori, T. Mori, G. Saito and S. Tanaka, J. Phys.: Condens. Matter 6 (1994) LA79.

[10] N. Harrison, A. House, I. Deckers, J. Caulfield, J. Singleton, F. Herlach, W. Hayes, M. Kurmoo and P. Day, Phys. Rev. B 52 (1995) 5584.

[11] D. Shoenberg Magnetic Oscillations in Metals (Cambridge University Press, Cambridge, 1984).

[12] T. Osada, A. Kawasumi, R. Yagi, S. Kagoshima, N. Miura, M. Oshima, H. Mori, T. Nakamura and G. Saito, Solid State Commun. 75 (1990) 901.

[13] P.S. Sandhu, G.J. Athas, J.S. Brooks, E.G. Haanappel, J.D. Goettee, D.W. Rickel, M. Tokumoto, N. Kinoshita, T. Kinoshita and Y. Tanaks, Phys. Rev. B, submitted for publication.

[14] V.N. Laukhin, N.D. Kusch, L.I. Buravov, A.G. Komenko, E.B. Yagubskii, H. Rakoto, J.M. Broto, L. Brossard, A.E. Kovalev, M.V. Kartsovnik, L.P. Rozenberg and R.P. Shibaeva, unpublished results.

[15] P. Christ, W. Biberacher, A.G.M. Janssen, M.V. Kartsovnik, A.E. Kovalev, N.D. Kusch, E. Steep and K. Andres, this conference.

[16] S.J. Blundell and J. Singleton, Phys. Rev. B 53 (1996) 5609.

[17] K. Yamaji, J. Phys. Soc. Jpn. 58 (1989) 1520.

[18] M.V. Kartsovnik, V.N. Laukhin, S.I. Pesotskii, L.F. Schegolev, and V.M. Yakovenko, J. Physique I2 (1993) 89.

[19] T. Osada, S. Kagoshima and N. Miurs, Phys. Rev. B 46 (1992) 1812.

[20] Y. Iye, R. Yagi, N. Hanasaki, S. Kagoshima, H. Mori, H. Fujimoto and G. Saito, J. Phys. Soc. Jpn. 63 (1994) 674.

[21] A. House, N. Harrison, I. Deckers, J. Caulfield, J. Singleton, F. Herlach, W. Hayes, M. Kurmoo and P. Day, Phys. Rev. B 53 (1996) 9127.

[22] M. Doporto, J. Singleton, F.L. Pratt, J. Caulfield, W. Hayes, J.A.A.J. Perenboom, I. Deckers, G. Pitsi, M. Kurmoo and P. Day, Phys. Rev. B 49 (1994) 3934.

[23] H. Goto and Y. Natsume, J. Phys. Soc. Jpn. 63 (1994) 1465. 